\title{
Incorporating double copies of a chromatin insulator into lentiviral vectors results in less viral integrants \\ Troels T Nielsen ${ }^{1,2}$, Johan Jakobsson ${ }^{1}$, Nina Rosenqvist ${ }^{1}$ and Cecilia Lundberg*1
}

\author{
Address: ${ }^{1}$ CNS Gene Therapy Unit, Wallenberg Neuroscience Center, Department of Experimental Medical Sciences, Lund University, BMC A11, \\ S-221 84 Lund, Sweden and 'Section of Neurogenetics, Department of Cellular and Molecular Medicine, Panum Instituttet, University of \\ Copenhagen, Copenhagen, Denmark \\ Email: Troels T Nielsen - troelsn@sund.ku.dk; Johan Jakobsson - Johan.Jakobsson@med.lu.se; Nina Rosenqvist - nros@lundbeck.com; \\ Cecilia Lundberg* - Cecilia.Lundberg@med.lu.se \\ * Corresponding author
}

Published: 24 February 2009

BMC Biotechnology 2009, 9:13 doi:10.1/86/1472-6750-9-13
Received: 18 September 2008

Accepted: 24 February 2009

This article is available from: http://www.biomedcentral.com/1472-6750/9//3

(c) 2009 Nielsen et al; licensee BioMed Central Ltd.

This is an Open Access article distributed under the terms of the Creative Commons Attribution License (http://creativecommons.org/licenses/by/2.0), which permits unrestricted use, distribution, and reproduction in any medium, provided the original work is properly cited.

\begin{abstract}
Background: Lentiviral vectors hold great promise as gene transfer vectors in gene therapeutic settings. However, problems related to the risk of insertional mutagenesis, transgene silencing and positional effects have stalled the use of such vectors in the clinic. Chromatin insulators are boundary elements that can prevent enhancer-promoter interactions, if placed between these elements, and protect transgene cassettes from silencing and positional effects. It has been suggested that insulators can improve the safety and performance of lentiviral vectors. Therefore insulators have been incorporated into lentiviral vectors in order to enhance their safety profile and improve transgene expression. Commonly such insulator vectors are produced at lower titers than control vectors thus limiting their potential use.

Results: In this study we cloned in tandem copies of the chicken $\beta$-globin insulator (cHS4) on both sides of the transgene cassette in order to enhance the insulating effect. Our insulator vectors were produced at significantly lower titers compared to control vectors, and we show that this reduction in titer is due to a block during the transduction process that appears after reverse transcription but before integration of the viral DNA. This non-integrated viral DNA could be detected by PCR and, importantly, prevented efficient transduction of target cells.

Conclusion: These results have importance for the future use of insulator sequences in lentiviral vectors and might limit the use of insulators in vectors for in vivo use. Therefore, a careful analysis of the optimal design must be performed before insulators are included into clinical lentiviral vectors.
\end{abstract}

\section{Background}

Lentiviruses are complex retroviruses that are capable of infecting non-dividing cells[1,2]. As a consequence lentiviral vectors, such as those based on HIV-1, efficiently transduce a variety of cell types including embryonic stem cells, haematopoietic cells and neurons, and have been suggested as candidate vectors for both in vivo and ex vivo gene therapy applications [3-5]. A key step in the retroviral life-cycle is integration of the provirus into the host genome. This integrating nature of retroviruses has been 
exploited in the development of gene transfer vectors and has been deemed essential for certain gene therapy strategies, including ex vivo gene transfer[6,7]. However, adverse issues related to the integration of a provirus such as insertional mutagenesis, promoter interference and positional effects have been highlighted by induction of malignancy in mouse models[8] and by the development of leukaemia in five patients in two clinical gene therapy trials $[9,10]$. Consequently, obtaining adequate safety and efficiency of viral vectors is a focus of interest in vector development.

Insertion of chromatin insulator elements into the vector sequence has been proposed as a solution to problems related to the integration of proviruses[11]. An insulator is a cis-acting element characterized by one or both of the following criteria: it prevents enhancer-promoter interactions, if placed in between these elements, and protects transgenic expression cassettes from silencing and positional effects $[11,12]$. Hence, if these characteristics could be transferred to a lentiviral vector it would confer protection from influences of the integrated viral sequence to surrounding endogenous genes, and since it may prevent silencing of a proportion of the proviruses, it may also enhance vector functionality allowing a lower number of integrants to be used in order to achieve therapeutic efficacy. Taken together, such improvements of the vector properties would be likely to reduce the risk related to the proviral integration[13].

However, before insulators are included into clinical gene therapy vectors, a number of issues remain to be clarified. So far, no studies have clearly demonstrated an enhanced safety of insulator vectors, although it has recently been shown that inclusion of insulators in lentiviral vectors suppresses clonal dominance in vitro and partially protects against genotoxicity and promoter interference [14-17]. Furthermore, improved transgene expression from insulator containing retro- and lentiviral vectors has been reported predominantly in cell lines of erythroid origin and appears to be cell-type dependent [18-20]. Finally, the inclusion of insulator-sequences has been reported to reduce the titer of viral vector preparations, which complicates transduction of difficult target cells or direct in vivo applications $[19,21,22]$.

In this study we addressed some of the issues of lentiviral vectors carrying chromatin insulators. For this we designed a set of insulator-lentiviral vectors that were tested in cells of different lineages. We found that lentiviral vectors are indeed capable of incorporating insulator sequences leading to efficient transgene expression in different cell types. However, a careful examination of their functionality suggests that the titer is compromised at a step that takes place after reverse transcription but before proviral integration. This finding has implications for the design of insulator-containing lentiviral vectors and highlights the importance of extensive studies of vector functionality following inclusion of cis-acting elements.

\section{Results \\ Lentiviral vectors}

We constructed second generation HIV-1 derived lentiviral vectors with the chicken $\beta$-globin insulator (cHS4) in various configurations (Figure 1). All vectors carried the central polypurine tract (CPPT) and the woodchuck hepatitis post-transcriptional regulatory element (WPRE), which have been shown to enhance the functionality of lentiviral vectors $[23,24]$. We used two promoters, the human cytomegalovirus promoter (CMV) and the human elongation factor $1 \alpha$ promoter (EF1 $\alpha$ ), in order to avoid misinterpretation of the results due to possible promoterspecific events. The first design of the insulator vectors had tandem copies of the 250 bp core element[25] placed just upstream of the expression cassette $(\mathrm{s} 2 \times 250 \mathrm{bp}$.CMV, figure $1 \mathrm{~B})$. The second design had a second doublet of the $250 \mathrm{bp}$ core element placed just after the transcriptional cassette $(\mathrm{d} 2 \times 250 \mathrm{bp}$, figure $1 \mathrm{C}$ and $1 \mathrm{~F})$. We also used our previously reported vector, which carries the full length $1.2 \mathrm{~kb}$ insulator into the 3'LTR $(1.2 \mathrm{~kb} . \mathrm{CMV}$, figure 1D)[21]. This design leads to a duplication of the insulator sequence into the $5^{\prime}$ LTR during reverse transcription. As control vectors we used CMV.SIN and EF1 $\alpha . S I N$ (Figure $1 \mathrm{~A}$ and $1 \mathrm{E})$.

\section{Titers of insulator-containing vectors are reduced}

Previously, we and others have suggested that the functional titer is reduced when insulator sequences are incorporated into retroviral and lentiviral vectors[19,21,22]. Therefore, we decided to estimate vector titer in two independent ways. The functional titer was estimated by transducing 293 T cells with limited dilutions of vector stocks. The proportion of GFP expressing cells was then estimated and transductions containing around 10\% GFP expressing cells were used to calculate a functional titer. A relative DNA titer was measured by quantifying the level of viral DNA relative to endogenous genomic DNA by performing real-time PCR on DNA from transduced 293T cells.

The vector carrying a single copy of the $2 \times 250$ bp doublet $(\mathrm{s} 2 \times 250 \mathrm{bp} . \mathrm{CMV})$ was produced at a similar functional titer as the control vectors (CMV.SIN and EF1 $\alpha . S I N$ ) (Table 1). However, the titer of our vector carrying the 1.2 $\mathrm{kb}(1.2 \mathrm{~kb} . \mathrm{CMV})$ insulator in the LTR was significantly reduced $(\mathrm{P}<0.01)$ more than 6 -fold (average: $4.9 \times 10^{7}$ $\mathrm{TU} / \mathrm{ml}, 1.2 \mathrm{~kb} . \mathrm{CMV}$ vector vs. $3.0 \times 10^{8} \mathrm{TU} / \mathrm{ml}, \mathrm{CMV} . \mathrm{SIN}$ ) which is in agreement with what we have reported previously with a similar design lacking the CPPT[21]. In addition, the functional titer of the CMV vector carrying double copies of the $2 \times 250$ bp element $(\mathrm{d} 2 \times 250$ 


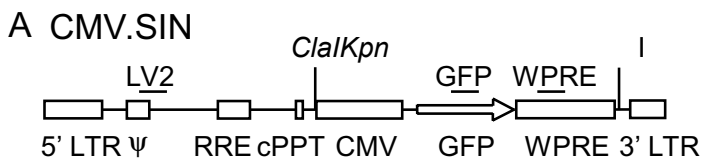

C d2x250bp.CMV

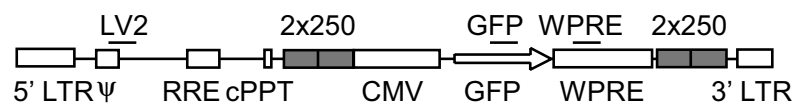

E EF $1 \alpha . S I N$

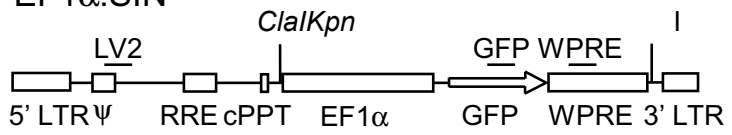

B s2x250bp.CMV

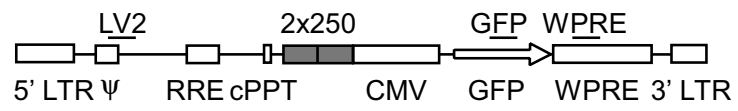

D $1.2 \mathrm{~kb} . \mathrm{CMV}$

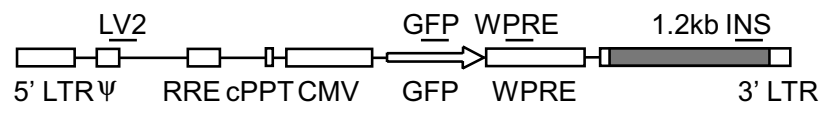

F d2x250bp.EF1 $\alpha$

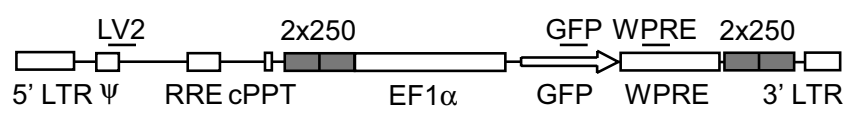

Figure I

Outline of lentiviral vectors. Schematic drawing of vectors used in this study. Vectors A-D utilize the CMV-promoter whereas vectors $\mathrm{E}-\mathrm{F}$ use the EFI $\alpha$-promoter. (A, E) Control vectors for the group of CMV and EFI $\alpha$ vectors respectively. Cloning sites for the cis-elements are shown. (B) One tandem repeat $(2 \times 250)$ of the core element of chicken $\beta$-globin insulator (cHS4) was inserted (sense orientation) prior to the promoter. (C, F) Two copies of the tandem repeat were inserted into the vectors - one just upstream the promoter and one just downstream the expression cassette. (D) The entire cHS4-insulator (I.2 kb) was inserted in the 3' LTR in sense orientation. (A-F) Horizontal lines denote the location of primers (labelled LV2, GFP, WPRE and INS) used for quantitative real-time PCR (qPCR). Legend: LTR - Long Terminal Repeat, $\psi$ - packaging signal, RRE - Rev Responsive Element, cPPT - Central Poly Purine Tract, CMV - Cytomegalo virus promoter, EFI $\alpha$ - Elongation Factor I $\alpha$ promoter, GFP - Green Fluorescent Protein, WPRE - Woodchuck Posttranscriptional Regulatory Element, $2 \times 250$ tandem repeat of the cHS4 core element and I.2 kb - full length cHS4 insulator sequence.

bp.CMV) was significantly $(\mathrm{P}<0.01)$ reduced almost 20 fold (average $1.6 \times 10^{7} \mathrm{TU} / \mathrm{ml}$ ) compared to the control vector (CMV.SIN). The same was true for the $\mathrm{d} 2 \times 250$ bp.EF1 $\alpha$ vector where the functional titer was reduced almost 28 -fold $\left(1.4^{*} 10^{7} \mathrm{TU} / \mathrm{ml}\right.$ of d2 $\times 250$ bp.EF1 $\alpha$ vs. $3.9 * 10^{8}$ of EF1 $\left.\alpha . S I N\right)$. Interestingly, such a dramatic reduction in the relative DNA titer could not be observed, where only the titer of $\mathrm{d} 2 \times 250 \mathrm{bp}$.EF1 $\alpha$ was significantly reduced (4-fold, $9.0^{*} 10^{7} \mathrm{TU} / \mathrm{ml}$ of $\mathrm{d} 2 \times 250 \mathrm{bp}$.EF1 $\alpha$ vs. $3.9^{*} 10^{8}$ of EF1 $\left.\alpha . S I N\right)$.
In order to analyse if the discrepancy between functional titer and relative DNA titer of $\mathrm{d} 2 \times 250 \mathrm{bp}$ and $1.2 \mathrm{~kb}$.CMV vectors was due to loss of the transcriptional cassette by homologous recombination during the transduction process, we transduced 293T cells with the four vectors carrying the CMV promoter (Figure 1A-D). DNA was harvested at 28 days post-transduction, and real-time PCR was performed using four sets of primers (LV2-, GFP-, WPRE- and INS-primers, see figure 1 for primer location) to determine the abundance of each of the different sequences in relation to each other. The LV2-primer was used as reference. At this late time-point post transduction

Table I: Titer of viral batches

\begin{tabular}{lll}
\hline Vector & $\begin{array}{l}\text { Functional titer } \\
* 10^{8} \mathbf{T U} / \mathbf{m l}\end{array}$ & Relative DNA titer $* 10^{8} \mathbf{U} / \mathbf{m l}$ \\
\hline CMV.SIN & $3.0 \pm 0.4$ & $\mathrm{~N} / \mathrm{A}$ \\
$\mathrm{s} 2 \times 250 . \mathrm{CMV}$ & $1.2 \pm 0.5$ & $1.6 \pm 0.05$ \\
$\mathrm{~d} 2 \times 250 . \mathrm{CMV}$ & $0.16 \pm 0.05 *$ & $0.62 \pm 0.05$ \\
$\mathrm{I} .2 \mathrm{~kb} . \mathrm{CMV}$ & $0.49 \pm 0.08 *$ & $1.2 \pm 0.3$ \\
EFI $\alpha . \mathrm{SIN}$ & $3.9 \pm 0.9$ & $\mathrm{~N} / \mathrm{A}$ \\
d2 $\times 250 . \mathrm{EFI} \alpha$ & $0.14 \pm 0.02 *$ & $0.9 \pm 0.2 *$ \\
\hline
\end{tabular}

Functional and relative DNA titer of viral vectors (mean \pm SEM). CMV.SIN and EFI $\alpha . S I N$ work as references for each group of vectors. By definition the relative DNA titers of the control vectors (CMV.SIN and EFI $\alpha . S I N$ ) are identical to their respective functional titers. * denotes significant $(P<0.05)$ different titer compared to the vector's respective control. 
we expected that potential episome-like structures would be diluted out due to cell division and that only properly integrated viral sequences would contribute to the realtime PCR readings. We found an approximate 1:1-ratio between LV2-, GFP- and WPRE-sequences in all vectors but the one carrying two copies of the insulator doublet (d2 $\times 250$ bp.CMV vector, Table 2$)$. The latter showed a significant $(P<0.001)$ decrease in the abundance of the GFP-sequence when compared to the control vector ( 0.69 $\pm 0.02, \mathrm{~d} 2 \times 250$ bp.CMV vs. $1.15 \pm 0.05$, CMV.SIN, relative units) along with an insignificant tendency that also the WPRE-sequence was underrepresented $(0.88 \pm 0.08$, $\mathrm{d} 2 \times 250$ bp vs. $1.25 \pm 0.09$, CMV.SIN, relative units). This suggests that only the $\mathrm{d} 2 \times 250$ bp vector loses the transcriptional cassette during transduction but that this happens only in a minority of integrants. Such rearrangements are common in recombinant retroviral vectors with direct repeats[26,27], and our data suggest that the same might be true for some configurations of direct repeats in lentiviral vectors. However, as only a minority of the $\mathrm{d} 2 \times 250 \mathrm{bp}$.CMV integrants seems to have undergone rearrangements and since the other three vectors, including the $1.2 \mathrm{~kb}$.CMV vector, were integrated in a proper configuration, rearrangements followed by loss of the transcriptional cassette do not seem to explain the discrepancy between the functional titer and the relative DNA titer. Finally, we found that the ratio between the QPCR readings of LV2 and INS was approximately 1:2 after transduction with the $1.2 \mathrm{~kb}$.CMV vector compared to $1: 1$ in plasmid DNA (Table 2), showing that the full length $1.2 \mathrm{~kb}$ insulator is efficiently duplicated during reverse transcription.

In summary, the two different ways of titration and the analysis of possible vector rearrangement suggest that the vectors containing two separated copies of the insulator in the reversed transcribed form $(\mathrm{d} 2 \times 250$ bp.EF1 $\alpha, \mathrm{d} 2 \times$ 250 bp.CMV and $1.2 \mathrm{~kb} . \mathrm{CMV}$ ) are fully functional and that the transgene expression from these vectors appears

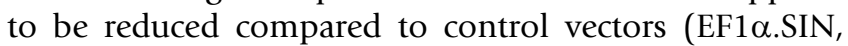
CMV.SIN and s2 $\times 250$ bp.CMV).

\section{Transgene expression}

To determine the influence of the insulator elements on transgene expression three different cell-types of different lineages were transduced with the lentiviral vectors at a multiplicity of infection (MOI) of 1 and 5 based on the relative DNA titer. The use of the relative DNA titer, which is not based on transgene expression capability during titration, allows comparison between different vector designs. For these experiments we used human 293T cells, which are derived from embryonic kidney, RN33B, which are immortalized rat neural precursor cells, and K562, which are human erythroid progenitor cells. The transduced cells were passaged for 7 days and then transgene expression (GFP) was determined by flow cytometry. The neural stem cell line, RN33B, was in a separate experiment also differentiated in vitro for 7 days.

When transducing the cell lines with the $\mathrm{d} 2 \times 250 \mathrm{bp}$ vectors, we found less GPF-positive cells and lower mean fluorescence (mean fluorescence units - MFU) independent of cell type and promoter choice when comparing with control vectors (Figure $2 \mathrm{~A}-\mathrm{Q}$ ), which suggests that the cHS4 core element fails to increase transgene expression. In addition, the insulator was unable to prevent transgene silencing during differentiation of RN33B cells (results not shown). This is in agreement with recent results showing that the $250 \mathrm{bp}$ core element of the cHS4-insulator is insufficient for insulating properties in a retroviral and lentiviral context[15,28]. Surprisingly, however, QPCR analyses of the proviral load in transduced cell cultures revealed significantly lower levels of viral DNA in cultures transduced with the $\mathrm{d} 2 \times 250$ bp vectors compared to control vectors (Figure $2, \mathrm{C}, \mathrm{H}, \mathrm{K}$ and $2 \mathrm{~N}$ ), despite the fact that we had normalized the various transductions using the

Table 2: Integrity of proviral integrants

\begin{tabular}{|c|c|c|c|c|}
\hline Vector & LV2 primers (Reference) & $\begin{array}{l}\text { Relative measurement } \\
\text { GFP primers }\end{array}$ & $\begin{array}{l}\text { Relative measurement } \\
\text { WPRE primers }\end{array}$ & $\begin{array}{l}\text { Relative measurement } \\
\text { INS primers }\end{array}$ \\
\hline CMV.SIN & I & $1.15 \pm 0.05$ & $1.25 \pm 0.09$ & - \\
\hline s2 $\times 250$ bp.CMV & 1 & $1.11 \pm 0.02$ & $1.39 \pm 0.14$ & - \\
\hline $\mathrm{d} 2 \times 250$ bp.CMV & I & $0.69 \pm 0.02 *$ & $0.88 \pm 0.08$ & - \\
\hline I.2 kb.CMV & I & $1.20 \pm 0.05$ & $1.21 \pm 0.29$ & $2.23 \pm 0.04 *$ \\
\hline I.2 kb.CMV (Plasmid) & I & - & - & $0.98 \pm 0.03$ \\
\hline
\end{tabular}

Real-time PCR data showing the abundance of four sequences (see figure I for primer location) present in the viral vectors. 293T cells were transduced at MOI I (functional titer) and after 28 days cells were harvested and analysed. Abundance of sequences detected with primer pairs GFP, WPRE and INS was calculated using LV2 primers as reference. For CMV.SIN and s2 $\times 250$ bp.CMV the ratio between LV2, GFP and WPRE was approximately $1: 1: 1$ as expected. With respect to the $\mathrm{d} 2 \times 250$ bp.CMV vector the ratio was roughly 1:0.7:0.9, which indicate that the GFP sequence is less abundant in cell cultures transduced with this vector $(P<0.00 I)$. This suggests that the vector undergoes some degree of rearrangement during production, reverse transcription or integration. In contrast, the $1.2 \mathrm{~kb}$.CMV vector did not show any sign of rearrangements, as the ratio of LV2, GFP, WPRE and INS was approximately I:I:I:2 when measuring on genomic DNA of transduced cells. In addition, the ratio between LV2 and INS was I:I when measuring on 1.2 kb.CMV plasmid DNA as expected. Significance is denoted by*. 

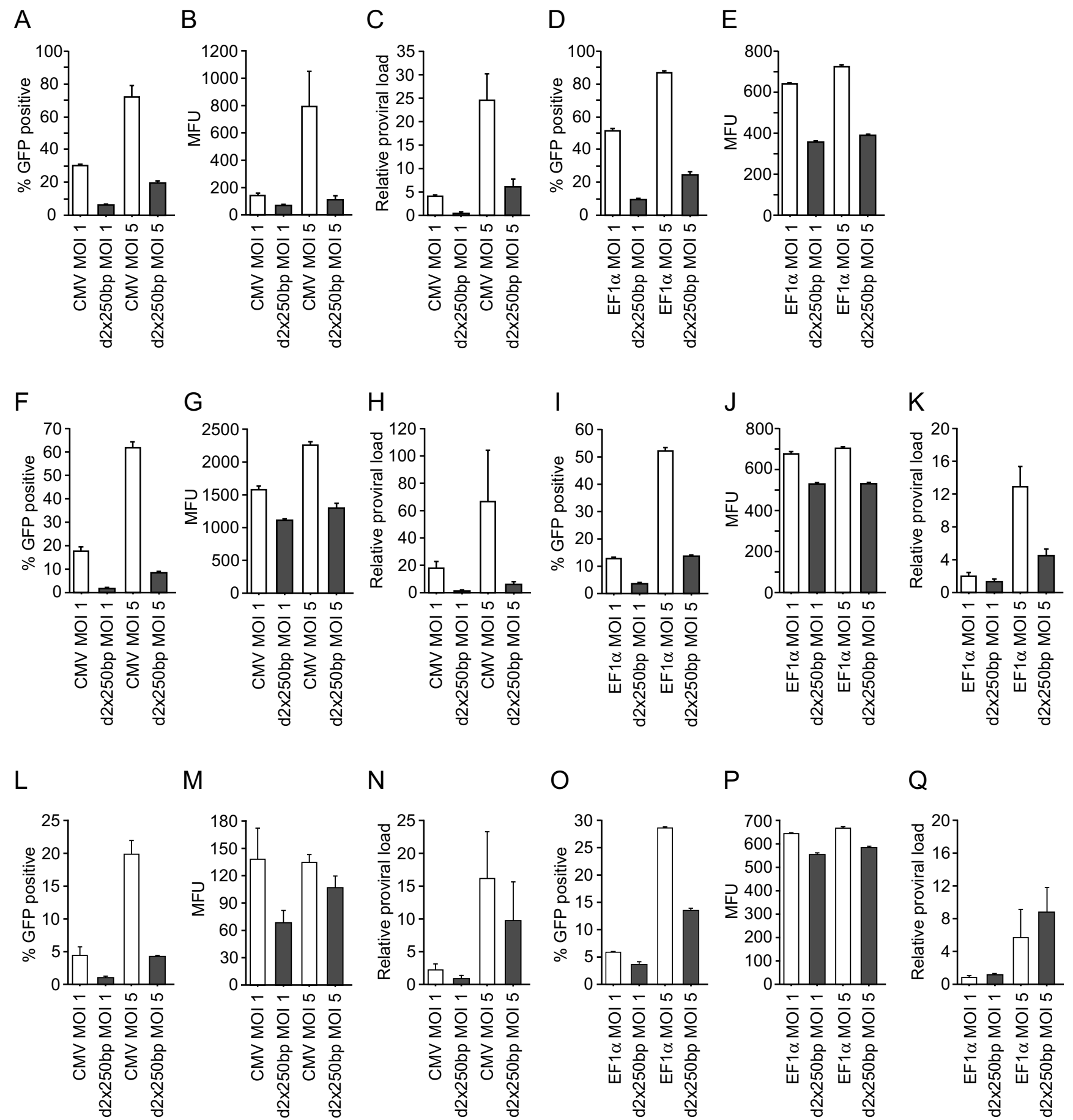

$\mathrm{P}$

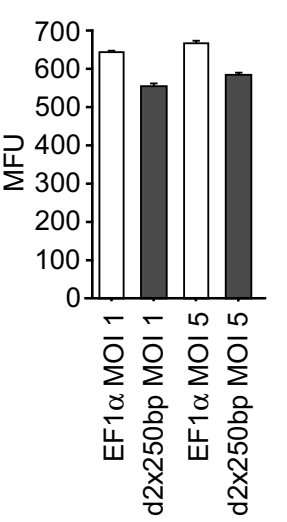

Q

Figure 2

Expression data of the $\mathbf{d} 2 \times \mathbf{2 5 0}$ bp vectors. Flow cytometric data and corresponding determination of proviral load for $\mathrm{d} 2 \times 250$ vectors in three different cell types: RN33B (naïve) (A-E), 293T (F-K) and K562 (L-Q). Cells were transduced at MOI $I$ and 5 (relative DNA titer) and analysed 7 days after transduction. The figure shows the percentage of GFP positive cells for each vector (A, D, F, I, L and $O$ ) along with the corresponding mean fluorescence (MFU) (B, E, G, J, M and P) and proviral load (the latter only for selected vectors) $(\mathrm{C}, \mathrm{H}, \mathrm{K}, \mathrm{N}$ and $\mathrm{Q})$. Error bars denote standard deviations. 
relative DNA titer. This difference in viral DNA accounted for most of the difference in transgene expression. This is surprising since we have previously used the relative DNA titer for titer determination of vectors with another commonly used cis-element, the scaffold attachment region (SAR) [19,29], and subsequently found complete agreement between the proviral loads of cell cultures transduced with the vector containing this cis-element and the control vector (supplementary information (Additional file 1 and 2: Supplementary information.pdf and Supplementary tables.pdf)). In addition, these data confirm that the negative effects on vector functionality observed when inserting the $2 \times 250$ bp doublet after the WPRE are not caused by the size increment of the vector, since the insertion of the SAR element, which is similar in size compared to the $2 \times 250$ bp doublet, in this position does not influence the functional titer, the relative DNA titer, transgene expression levels or proviral loads of transduced cultures. Thus the reduced transgene expression from the $\mathrm{d} 2 \times 250$ bp vectors probably reflects impaired vector functionality with regard to cell entry, integration etc, and this might explain the observed discrepancies between the functional titer and the relative DNA titer.

Double copies of insulators result in less proviral integrants One explanation of the overestimation of titer when using relative DNA titration of the $\mathrm{d} 2 \times 250 \mathrm{bp}$ and $1.2 \mathrm{~kb}$.CMV vectors could be that these configurations of insulator sequences have a deleterious effect on vector functionality at a stage after reverse transcription (i.e. viral integration). Using real-time PCR to quantify viral DNA detects not only viral DNA that is properly integrated but also viral DNA that remains non-integrated. If such non-integrated viral DNA was abundant in cell cultures transduced with the $\mathrm{d} 2 \times 250 \mathrm{bp}$ and the $1.2 \mathrm{~kb}$.CMV vectors, titer determination using real-time PCR 3 days after transduction would overestimate the titers, compared to titer determination conducted 7 days post-transduction, where nonintegrated viral DNA will be more diluted due to ongoing cell division. In this study titer determination was performed 3 days post transduction where all viral integration has normally occurred[30], whereas viral DNA levels in the expression analyses were measured 7 days post transduction. A similar overestimation of the functional titer is unlikely to happen if the non-integrated viral DNA is present in a form not suitable for transgene expression, since only properly integrated and transgene expressing vector genomes will contribute to this titer determination. These circumstances could explain the inconsistent relationship between the relative and the functional titer and the proviral loads measured in the expression analyses of the $\mathrm{d} 2 \times 250 \mathrm{bp}$ and the $1.2 \mathrm{~kb}$.CMV vectors.

To test this hypothesis we transduced 293T cells at MOI 1 (based on functional titer) with the four CMV vectors.
Cells were passaged and part of each cell culture was harvested at days 3, 6, 14 and 27. Real-time PCR (using primers LV2 and ALB) on DNA isolated from these cells revealed that the proviral load was significantly $(\mathrm{P}<$ 0.001 ) decreased in cells transduced with the $\mathrm{d} 2 \times 250 \mathrm{bp}$ vector and the $1.2 \mathrm{~kb}$ vector during day 3-6 (on day six the proviral loads of the $\mathrm{d} 2 \times 250 \mathrm{bp}$ vector and the $1.2 \mathrm{~kb}$ vectors were $28 \% \pm 10 \%$ and $37 \% \pm 16 \%$, respectively, of their initial proviral loads measured on day three) (Figure $3)$. After the initial drop the proviral load stayed a constant low level for at least 27 days (only 3, 6 and 14 days time points are shown in the figure). In vectors containing none or only one insulator-doublet in the reverse transcribed form (CMV.SIN and s2 × 250 bp.CMV) no such drop was observed (Figure 3 ). These results support the hypothesis that lentiviral vectors carrying two separated copies of the cHS4 insulator in the reverse transcribed form are capable of efficient cell-entry and subsequent reverse transcription (since high levels of viral DNA can be detected 3 days post transduction), but that the transduction process is impaired at some step after reverse transcription but before integration (since the abundance of viral DNA declines during day 3-6 post transduction). Notably, the insulator sequence itself is not deleterious to the vector since we could not detect any decrease in viral load of the s $2 \times 250$ bp.CMV vector from day 3 to 6 (Figure 3$)$. The same was true for our vector containing the SAR element confirming that loss of viral DNA from day 3-6 was not attributable to the size increment of the $\mathrm{d} 2 \times$ 250 bp vector (supplementary figure 3 (Additional file 3:

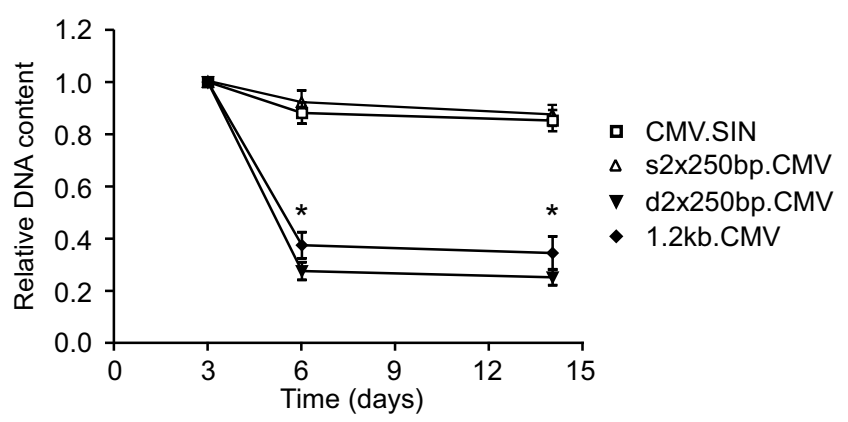

\section{Figure 3}

Persistence of viral DNA in transduced cells during a 14 day time period. 293T cells were transduced at MOI I (relative DNA titer) with the four vectors utilizing the CMVpromoter. After 3, 6, 14 and 27 days cells were harvested and the proviral load determined by QPCR using primers LV2 and ALB. The vectors containing two separated copies of the insulator ( $\mathrm{d} 2 \times 250$ bp.CMV and I.2 kb.CMV) elicit a significant $(*, p<0.05)$ drop in proviral load within the first 6 days after transduction compared to control vectors, CMV.SIN and s2 $\times 250 \mathrm{bp} . \mathrm{CMV}$. The experiment was continued for 27 days with no change in proviral load compared to the 14 days time point. Error bars denote standard error of the mean. 
Supplementary figures.pdf)). It is possible that two identical insulator sequences separated by an appropriate spacing as in the $\mathrm{d} 2 \times 250 \mathrm{bp}$.CMV vector and the reversed transcribed form of the $1.2 \mathrm{~kb}$.CMV vector induce the formation of secondary structure incompatible with proper transduction and transgene expression.

\section{Non-integrating vectors prevent efficient transduction}

Our results with the $\mathrm{d} 2 \times 250$ bp vectors suggest that the low titer is due to several independent defects. As shown above, a large proportion of the vectors are unable to integrate and express the transgene. Furthermore, a minor proportion of the vectors that integrate will have lost the transgene cassette due to vector rearrangements. However, it is worth pointing out that in contrast to oncoretroviral vectors with a similar design functional particles are produced[22].

To test the transduction potential of these remaining functional vectors, we transduced RN33B cells at an MOI of 1 and 5, this time based on the functional titers. At 7 days after transduction cells were harvested and flow cytometry performed. For the CMV vectors at a MOI of 1 , the $\mathrm{d} 2 \times$ $250 \mathrm{bp}$ vector seemed to perform as well as the control vector (Figure $4 \mathrm{~A}$ and $4 \mathrm{~B}$ ). However, at high MOI, the $\mathrm{d} 2$ $\times 250$ bp vector expressed GFP at a much lower level than the control vector (Figure 4A and 4B). When using EF1 $\alpha$ vectors the performance of the $\mathrm{d} 2 \times 250 \mathrm{bp}$ vector was even worse compared to the control vector (Figure $4 \mathrm{C}$ and $4 \mathrm{D})$.

We then transduced the neural stem cell line RN33B with increasing doses of lentiviral vectors (CMV.SIN, d2 × 250 bp.CMV and $1.2 \mathrm{~kb} . \mathrm{CMV}$ ) based on functional titer. At low MOIs the difference in expression between control vectors and insulator vectors was relatively small both with regard to the number of GFP positive cells and the fluorescence intensity (Figure 4E-F). However, at higher MOIs the dose-response curve of the insulator vectors reached plateau at a lower number of transduced cells and lower fluorescence intensity (Figure 4E-F). As to why this reduced expression is due to a block in the transduction pathway remains uncertain, but nonetheless efficient transduction and high levels of transgene expression from these vectors seem difficult to achieve.

\section{Discussion}

The original intention of this study was to analyse the effects of a chromatin insulator on transgene expression when inserted into lentiviral vectors. The idea was to use a set-up that was independent of any type of selection and that would allow us to study potential insulator effects in cells with multiple integrants. Up to date most studies using insulator sequences in retroviral or lentiviral settings have relied on drug selection of transgene expressing cells[19,28,31]. Furthermore, most studies have been designed so that only cells with single copy integrants have been analysed $[18,19,32,33]$. Such settings have little in common with clinical gene therapy protocols. In order to perform a non-selection based experiment we decided to titer the vectors using real-time PCR on DNA from transduced cells. This protocol has the advantage that it does not rely on transgene expression in order to quantify the titer of the vector preparation and allows a non-biased quantification of integrating particles. When using standard lentiviral vectors without or with only one insulator element we found highly reproducible results with this non-selection based strategy. However, when we tried to apply our non-biased strategy onto vectors containing two separated copies of the cHS4 insulator in the reversed transcribed form, we found that the DNA titration led to overestimation of the titer, supposedly because of the formation of secondary structure that is incompatible with efficient transduction. This block appears to take place in a step during transduction that is after reverse transcription but before integration. How this block of integration is established is unknown but it is possible that potential secondary structures, caused by the double copies of the insulator, impede proper formation and function of the pre-integration complex (PIC) e.g. by preventing correct alignment of the integrase to the integrase binding sites or by hampering proper trafficking of the viral genome to the nucleus after infection. However, several recent reports demonstrate efficient transgene expression from non-integrating lentiviral vectors present as classical 1-LTR or 2LTR circles[34,35]. These studies describe long-term expression in post-mitotic cells at levels comparable to integrating vectors. Since we show reduced expression from double copy insulator vectors, the non-integrated vector copies that we demonstrate must exist in a different form incompatible with transgene expression.

These results have important consequences for the use of insulator sequences in lentiviral vectors. It has been suggested that insulators need to be present in double or more copies in order to be fully functional[36,37]. This has led to the suggestion that insulators form a loop-structure that is resistant to influences from surrounding chromatin. However, lentiviral and retroviral vectors carrying such double copies have been reported by several groups to be produced in low titer[15,19-22]. As we demonstrate in this study that phenomenon is due to a block during the infectious process, and, importantly, this seems to prevent efficient transduction. When target cells were transduced with increasing doses of vector, the resulting doseresponse curve reached a plateau at a lower number of transduced cells and with a markedly reduced mean fluorescence compared to control vectors. This suggests that efficient transduction of difficult target cells such as primary cells will be compromised with this kind of insula- 

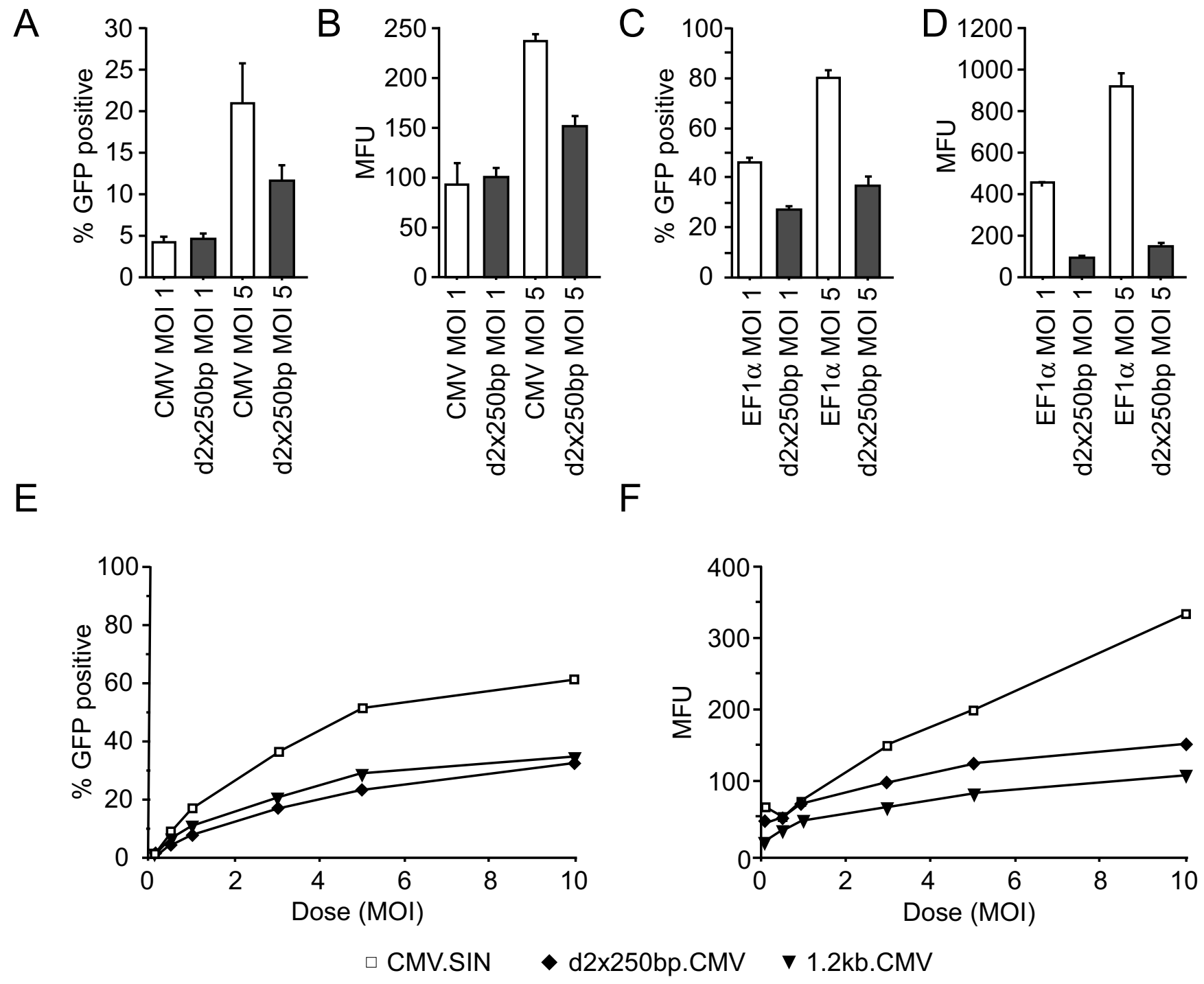

Figure 4

Expression data of the $\mathbf{d} \mathbf{2} \times \mathbf{2 5 0}$ bp vectors. (A-D) Flow cytometric data for the transduction of naïve RN33B cells with $\mathrm{d} 2 \times 250 \mathrm{bp}$ vectors and controls at $\mathrm{MOI} \mathrm{I}$ and 5 (functional titer). Cells were analysed 7 days after transduction. The percentage of GFP-positive cells is shown (A and C) along with the mean fluorescence (MFU) (B and D). Error bars denote standard deviations. E-F: Dose-response curves showing CMV.SIN, d2 $\times 250$ bp.CMV and I.2 kb.CMV vector performance in naïve RN33B cells. The percentage of GFP positive cells (E) and mean fluorescence is shown (F).

tor vectors, which will hamper their use in both ex vivo and in vivo gene therapy settings.

We and others have seen only a modest effect on transgene expression when using insulators in lentiviral vectors $[14,19,21]$. On the contrary, there appears to be strong positive effects when using more simple retroviral vectors such as those based on MLV $[18,28,33]$, and it is likely that the effects of an insulator will be dependent on the context, i.e. MLV contra lentiviral vectors. MLV-based and lentiviral vectors have different integration patterns. While MLV vectors preferentially integrate near the promoter region, the HIV-based vectors appear to integrate into the entire intragenic region[38,39]. It is not certain how these differences in integration sites affect transgene expression or the appearance of insertional mutagenesis. However, recent experiments in a tumorigenic mouse model show that self-inactivating lentiviral vectors have low genotoxicity compared to LTR-driven MLV vectors suggesting an enhanced safety profile of such lentiviral vectors[40]. Furthermore, lentiviral vectors appear to escape transgenic silencing more efficiently than MLV vectors. An example of this is the establishment of transgenic animals using lentiviral vectors[41], although several examples of lenti- 
viral silencing in the generation of transgenic animals have also been reported[42].

Other cis-elements that have successfully been used to enhance transgene expression from lentiviral vectors have been identified, e.g. scaffold or matrix attachment regions (SAR, MAR or S/MARs). These are A+T rich elements that have been suggested to be involved in the anchoring of the chromatin to the nuclear scaffold resulting in the formation of chromatin loops thereby avoiding gene silencing $[43,44]$, and elements that have shown to prevent transgene silencing in a lentiviral context have been derived from the human interferon- $\beta$ gene, the human immunoglobulin $\mu$ heavy-chain locus, the immunoglobulin $\kappa$, etc $[19,45,46]$. Furthermore, the use of S/MAR in combination with insulators have been shown to be highly effective in enhancing transgene expression from lentiviral vectors [19,47]. However, the effect of the S/MAR elements appears, in most cases, to be tissue specific (for review see[48]), which limits their general application, and identification of S/MAR elements active in neural cells will be important for gene therapeutic applications in the brain. Improving lentiviral vector characteristics with insulators or other cis-elements is therefore still an area of interest.

\section{Conclusion}

In conclusion our results demonstrate that insulator sequences can be incorporated into lentiviral vectors either in the LTR or flanking the transgene cassette. However, when double copies are used these vectors have a reduced functionality due to a block during the infectious process, and consequently such vectors transduce target cells poorly. Therefore, a careful analysis of the optimal design must be performed before insulators are included into clinical lentiviral vectors. Furthermore, it still remains to be proven that insulator sequences actually improve the safety profile of lentiviral vectors.

\section{Methods}

\section{Transfer plasmids}

The 250 bp core element of the cHS4 insulator was amplified from plasmid pJC5-4 (kindly provided by G. Felsenfeld, Laboratory of Molecular Biology, National Institute of Diabetes and Digestive Kidney Diseases, National Institute of Health, Bethesda) by four PCR-reactions using the following PCR-reaction: $3 \mathrm{~min}$ at $95^{\circ} \mathrm{C}$ followed by 30 cycles of $30 \mathrm{sec}$ at $95^{\circ} \mathrm{C}, 45 \mathrm{sec}$ at $55^{\circ} \mathrm{C}$ and $90 \mathrm{sec}$ at $72^{\circ} \mathrm{C}$. Primers were designed according to Chung et al.[25] and contained additional restriction sites (ClaI, KpnI and MluI) making it possible to join two 250 bp core elements into a $500 \mathrm{bp}$ doublet where each core element was situated in the same direction. Primers were as follows: Kpn up: 5'-GGT ACC GGA GCT CAC GGG GAC AGC-3' Kpn down: 5'-GGT ACC CCT AAA GCT TTT TCC
CCG TA-3' Cla up: 5'-ATC GAT GGA GCT CAC GGG GAC AGC-3' Cla down: 5'-ATC GAT CCT AAA GCT TTT TCC CCG TA-3' Mlu up: 5'-ACG CGT GGA GCT CAC GGG GAC AGC-3' and Mlu down: 5'-ACG CGT CCT AAA GCT TTT TCC CCG TA-3'. Two doublets were generated: One was flanked by KpnI-sites whereas the other was flanked by ClaI-sites. These two fragments were then cloned in the sense orientation into the ClaI and the KpnI-sites of pHRCMV.GFP.W (CMV.SIN) (kind gift from D. Trono, Lausanne, Switzerland) and pHR-EF1 $\alpha . G F P . W$ (EF1 $\alpha . S I N)$ (kindly provided by N.B. Woods, Lund, Sweden) using standard cloning procedures to generate pHR$2 \times 250$ bp.CMV.GFP.W. $2 \times 250(\mathrm{~d} 2 \times 250$ bp.CMV $)$ and pHR-2 $\times 250$ bp.EF1a.GFP.W. $2 \times 250$ (d2 $\times 250$ bp.EF1 $\alpha$ ). In addition a vector containing only one insulator doublet was constructed (pHR-2 × 250.CMV.GFP.W, s2 $\times 250$ bp.CMV). The vector pHR-CMV.GFP.W.1.2 kb (1.2 kb.CMV) has been described elsewhere and was only modified by the addition of the cPPT fragment[21].

Lentiviral vectors were produced as described previously[4]. Briefly, transfer plasmids were co-transfected with pMD.G and pBR $\Delta 8.91$ into 293T-cells, supernatants were collected on days 3 and 4 and concentrated by ultracentrifugation. Viral vector preparations were not treated with DNAse during the production step, since this has been shown not to influence the functional titer or the relative DNA titers[49] (and unpublished observations). We also excluded different levels of contaminating plasmid DNA in the various vector preparations by performing real-time PCR directly on concentrated viral vector stocks and found no difference between the plasmid content of control vectors compared to insulator vectors $(\mathrm{P}=0.52$, data not shown). All results were reproduced with several independently produced viral batches.

\section{Determination of viral titer}

Functional titers of vector preparation were assessed by transduction of 100,000 cells (293T) with serial dilutions of vector stocks. A dilution resulting in less than $10 \%$ GFP-positive cells was used for determining the functional titer of each vector. Each vector was titrated against the control vectors CMV.SIN or EF1 $\alpha$.SIN.

Relative DNA titers were assessed by transduction of 50,000 cells $(293 \mathrm{~T})$ with serial dilutions of vector stocks. After $72 \mathrm{~h}$ cells were collected and lysed and subjected to Taqman PCR[21] using the following primers LV2-F: 5'ACT TGA AAG CGA AAG GGA AAC-3', LV2-R: 5'-CAC CCA TCT CTC TCC TTC TAG CC-3' LV2-Probe: 5'-FamAGC TCT CTC GAC GCA GGA CTC GGC-Tamra-3'. All samples were analysed in triplicates. Using the comparative $\mathrm{C}_{\mathrm{T}}$-method (userbulletin\#2, http://www.appliedbio systems.com) the relative DNA-titers were calculated in relation to the control vectors, CMV.SIN and EF1 $\alpha$.SIN. By 
definition the relative DNA-titer of CMV.SIN and EF1 $\alpha . S I N$ equalled their determined functional titer.

\section{Cell culturing, transduction, flow cytometric analyses and determination of proviral load}

293T cells and $\mathrm{K} 562$ were cultured at $37^{\circ} \mathrm{C}$ in humidified atmosphere and 5\% $\mathrm{CO}_{2}$ in DMEM and RPMI 1640 respectively. Media was supplemented with L-glutamine and $10 \%$ foetal bovine serum. RN33B and HiB5 cells were cultured and differentiated as described elsewhere[50].

Cells were transduced simply by applying the appropriate amount of viral stock solution directly to the media of cell cultures.

Flow cytometric analyses were performed as described by Jakobsson et al[21]. Proviral loads of analysed cell cultures were determined by real-time PCR. Briefly, cells were harvested and lysed in $18 \mu \mathrm{l} 50 \mathrm{mM}$ Tris-HCl, $\mathrm{pH} 8.2$ (Amresco), $100 \mathrm{mM} \mathrm{NaCl}$ (Merck), $5 \mathrm{mM}$ EDTA (Chemicon) and $0.5 \%$ SDS (Chemicon). After addition of $2 \mu \mathrm{l}$ proteinase $\mathrm{K}(10 \mathrm{mg} / \mathrm{ml}$, Gibco BRL) samples were incubated at $55^{\circ} \mathrm{C}$ for 30 minutes. $200 \mu$ of water was added and samples were boiled for 10 minutes. Proviral load was assessed by real-time PCR using the LV2 primer (above) in reference to endogenous genes (the IL-2 gene in rat cells and the albumin, ALB, gene in human cells). Sequences of the IL-2 and albumin primers are: IL-2 Forward, 5'-GCC TTG TGT GTT ATA AGT AGG AGG C-3', IL-2 Reverse, 5'AGT GCC AAT TCG ATG ATG AGC-3', IL-2 Probe, 5'-FamTCT CCT CAG AAA TTC CAC CAC AGT TGC TG-Tamra-3', ALB Forward, 5'-TGA AAC ATA CGT TCC CAA AGA GTT T-3', ALB Reverse, 5'-CTC TCC TTC TCA GAA AGT GTG CAT AT-3' and ALB Probe, 5'-Fam-TGC TGA AAC ATT CAC CTT CCA TGC AGA-Tamra-3'.

\section{Testing vector integrity}

Quantitative real-time PCR was performed on cell lysates 28 days after infection as described above. Primers targeting a viral sequence (LV2, see above), GFP, WPRE and 1.2 $\mathrm{kb}$ insulator (INS) were used. Sequences of primers and probes were: GFP Forward, 5'-ACT ACA ACA GCC ACA ACG TCT ATA TCA-3, GFP Reverse, 5'-GGC GGA TCT TGA AGT TCA CC-3', GFP Probe, 5'-Fam-CGA CCA GCA GAA GAA CGG CAT CA-Tamra-3', WPRE Forward, 5'-CCG TTG TCA GGC AAC GTG-3', WPRE Reverse, 5'-AGC TGA CAG GTG GTG GCA AT-3', WPRE Probe, 5'-Fam-TGC TGA CGC AAC CCC CAC TGG T-Tamra-3', INS Forward, 5'ACC GCT CTT TGG AGA AGG TAA A-3', INS Reverse, 5'ATG AGA GAT AAT GGC CTT ACG TTG T-3', and INS Probe, 5'-Fam-CTT GCT AAA TCC AGC CCG ACC CTC CTamra-3'. Standard curves for each primer pair were constructed and the abundance of each sequence was calculated using the comparative $\mathrm{Ct}$ method, taking into account the efficiency of each primer set according to
Pfaffl (2001)[51]. All primer pairs used for real-time PCR were validated and showed efficiencies above $90 \%$.

\section{Statistics}

All experiments were performed in triplicate and repeated in two independent experimental rounds and with independently produced viral batches. Flow cytometry and qPCR analyses were subjected to analysis of variance (ANOVA) using SAS software followed by Tukey's HSD when appropriate.

\section{Authors' contributions}

TTN and JJ carried out the experiments, analysed data, and were primarily responsible for the preparation of the manuscript. NR carried out some of the cell culture work and data analyses. CL led the project, initiated the project and helped in writing the manuscript. All authors read and approved the final manuscript.

\section{Additional material}

\section{Additional file 1 \\ Supplementary information. Information on cloning and testing of a lentiviral vector containing the scaffold attachment region (SAR) of the human interferon- $\beta$ gene. \\ Click here for file \\ [http://www.biomedcentral.com/content/supplementary/1472- \\ 6750-9-13-S1.pdf] \\ Additional file 2 \\ Supplementary tables. Tables showing results from the titration of the SAR-containing vectors and results from the analyses of the proviral loads in cell cultures transduced with SAR-containing vectors. \\ Click here for file \\ [http://www.biomedcentral.com/content/supplementary/1472- 6750-9-13-S2.pdf] \\ Additional file 3 \\ Supplementary figures. Figures showing the SAR-containing vector and results from testing the vector. \\ Click here for file \\ [http://www.biomedcentral.com/content/supplementary/1472- 6750-9-13-S3.pdf]}

\section{Acknowledgements}

The authors would like to thank J. Bode and G. Felsenfeld for providing the SAR-plasmid and the insulator-plasmid, respectively, and we would like to thank $D$. Trono for providing the lentiviral vector system. In addition we would like to thank Anna-Karin Oldén, Birtgit Haraldsson, Anneli Josefsson and Ulla Jarl for technical assistance. This study was supported by the Swedish Research Council (grant \#13479), The Segerfalk and Crafoord Foundations.

\section{References}

I. Weinberg JB, Matthews TJ, Cullen BR, Malim MH: Productive human immunodeficiency virus type I (HIV-I) infection of 
nonproliferating human monocytes. I Exp Med |99|, 174: |477-|482

2. Naldini $L:$ Lentiviruses as gene transfer agents for delivery to non-dividing cells. Curr Opin Biotechnol 1998, 9:457-463.

3. Hamaguchi I, Woods NB, Panagopoulos I, Andersson E, Mikkola H, Fahlman C, Zufferey R, Carlsson L, Trono D, Karlsson S: Lentivirus vector gene expression during ES cell-derived hematopoietic development in vitro. J Virol 2000, 74:10778-10784.

4. Zufferey R, Nagy D, Mandel RJ, Naldini L, Trono D: Multiply attenuated lentiviral vector achieves efficient gene delivery in vivo. Nat Biotechnol 1997, 15:87|-875.

5. Uchida N, Sutton RE, Friera AM, He D, Reitsma MJ, Chang WC, Veres G, Scollay R, Weissman IL: HIV, but not murine leukemia virus, vectors mediate high efficiency gene transfer into freshly isolated G0/G I human hematopoietic stem cells. Proc Natl Acad Sci USA 1998, 95: I 1939-1 1944.

6. Buchschacher GL Jr, Wong-Staal F: Development of lentiviral vectors for gene therapy for human diseases. Blood 2000, 95:2499-2504.

7. Verma IM, Weitzman MD: Gene therapy: twenty-first century medicine. Annu Rev Biochem 2005, 74:7I I-738.

8. Li Z, Dullmann J, Schiedlmeier B, Schmidt M, Von KC, Meyer J, Forster M, Stocking C, Wahlers A, Frank O, Ostertag W, Kuhlcke K, Eckert HG, Fehse B, Baum C: Murine leukemia induced by retroviral gene marking. Science 2002, 296:497.

9. Hacein-Bey-Abina S, Garrigue A, Wang GP, Soulier J, Lim A, Morillon E, Clappier E, Caccavelli L, Delabesse E, Beldjord K, Asnafi V, Macintyre E, Dal CL, Radford I, Brousse N, Sigaux F, Moshous D, Hauer J, Borkhardt A, Belohradsky BH, Wintergerst U, Velez MC, Leiva L, Sorensen R, Wulffraat N, Blanche S, Bushman FD, Fischer A, Cavazzana-Calvo $M$ : Insertional oncogenesis in 4 patients after retrovirus-mediated gene therapy of SCID-XI. J Clin Invest 2008 I I 8:3 | 32-3142.

10. UCL Institute of Child Health, Great Ormond Street Hospital for Children NHS Trust: GOSH announces leukaemia case following gene therapy for X-SCID. 2007 [http://www.ich.ucl.ac.uk/ pressoffice/pressrelease 0059I]

II. Pikaart MJ, Recillas-Targa F, Felsenfeld G: Loss of transcriptional activity of a transgene is accompanied by DNA methylation and histone deacetylation and is prevented by insulators. Genes Dev 1998, 1 2:2852-2862.

12. Baum C, Dullmann J, Li Z, Fehse B, Meyer J, Williams DA, Von KC Side effects of retroviral gene transfer into hematopoietic stem cells. Blood 2003, 101:2099-21 I4.

13. Sinn PL Sauter SL, McCray PB Jr: Gene therapy progress and prospects: development of improved lentiviral and retroviral vectors - design, biosafety, and production. Gene Ther 2005 I 2:1089-1098.

14. Evans-Galea MV, Wielgosz MM, Hanawa H, Srivastava DK, Nienhuis AW: Suppression of clonal dominance in cultured human lymphoid cells by addition of the cHS4 insulator to a lentiviral vector. Mol Ther 2007, 15:80I-809.

15. Zychlinski D, Schambach A, Modlich U, Maetzig T, Meyer J, Grassman E, Mishra A, Baum C: Physiological promoters reduce the genotoxic risk of integrating gene vectors. Mol Ther 2008 16:7|8-725.

16. Robert-Richard E, Richard E, Malik P, Ged C, de VH, Moreau-Gaudry F: Murine retroviral but not human cellular promoters induce in vivo erythroid-specific deregulation that can be partially prevented by insulators. Mol Ther 2007, 15:173-182.

17. Steinwaerder DS, Lieber A: Insulation from viral transcriptional regulatory elements improves inducible transgene expression from adenovirus vectors in vitro and in vivo. Gene Ther 2000, 7:556-567.

18. Rivella S, Callegari JA, May C, Tan CW, Sadelain M: The cHS4 insulator increases the probability of retroviral expression at random chromosomal integration sites. I Virol 2000, 74:4679-4687.

19. Ramezani A, Hawley TS, Hawley RG: Performance- and safetyenhanced lentiviral vectors containing the human interferon-beta scaffold attachment region and the chicken betaglobin insulator. Blood 2003, 101:4717-4724.

20. Emery DW, Yannaki E, Tubb J, Nishino T, Li Q, Stamatoyannopoulos G: Development of virus vectors for gene therapy of beta chain hemoglobinopathies: flanking with a chromatin insula- tor reduces gamma-globin gene silencing in vivo. Blood 2002, 100:2012-2019.

21. Jakobsson J, Rosenqvist N, Thompson L, Barraud P, Lundberg C: Dynamics of transgene expression in a neural stem cell line transduced with lentiviral vectors incorporating the cHS4 insulator. Experimental Cell Research 2004, 298:6 I I-623.

22. Yao S, Osborne CS, Bharadwaj RR, Pasceri P, Sukonnik T, Pannell D, Recillas-Targa F, West AG, Ellis J: Retrovirus silencer blocking by the cHS4 insulator is CTCF independent. Nucleic Acids Res 2003, 31:5317-5323.

23. Follenzi A, Ailles LE, Bakovic S, Geuna M, Naldini L: Gene transfer by lentiviral vectors is limited by nuclear translocation and rescued by HIV-I pol sequences. Nat Genet 2000, 25:2 17-222.

24. Zufferey R, Donello JE, Trono D, Hope TJ: Woodchuck hepatitis virus posttranscriptional regulatory element enhances expression of transgenes delivered by retroviral vectors. J Virol 1999, 73:2886-2892.

25. Chung JH, Bell AC, Felsenfeld G: Characterization of the chicken beta-globin insulator. Proc Natl Acad Sci USA 1997, 94:575-580.

26. Jonsson J], Habel DE, Mclvor RS: Retrovirus-mediated transduction of an engineered intron-containing purine nucleoside phosphorylase gene. Hum Gene Ther 1995, 6:6II-623.

27. Pathak VK, Temin HM: Broad spectrum of in vivo forward mutations, hypermutations, and mutational hotspots in a retroviral shuttle vector after a single replication cycle: deletions and deletions with insertions. Proc Natl Acad Sci USA 1990 87:6024-6028.

28. Aker M, Tubb J, Groth AC, Bukovsky AA, Bell AC, Felsenfeld G, Kiem HP, Stamatoyannopoulos G, Emery DW: Extended core sequences from the cHS4 insulator are necessary for protecting retroviral vectors from silencing position effects. Hum Gene Ther 2007, I 8:333-343.

29. Mielke C, Kohwi Y, Kohwi-Shigematsu T, Bode J: Hierarchical binding of DNA fragments derived from scaffold-attached regions: correlation of properties in vitro and function in vivo. Biochemistry 1990, 29:7475-7485.

30. Brussel A, Sonigo P: Analysis of early human immunodeficiency virus type I DNA synthesis by use of a new sensitive assay for quantifying integrated provirus. J Virol 2003, 77:10119-10124.

31. Arumugam PI, Scholes J, Perelman N, Xia P, Yee JK, Malik P. Improved human beta-globin expression from self-inactivating lentiviral vectors carrying the chicken hypersensitive site-4 (cHS4) insulator element. Mol Ther 2007, I5: |863-I87| .

32. Emery DW, Yannaki E, Tubb J, Stamatoyannopoulos G: A chromatin insulator protects retrovirus vectors from chromosomal position effects. Proc Natl Acad Sci USA 2000, 97:9150-9155.

33. Li CL, Emery DW: The cHS4 chromatin insulator reduces gammaretroviral vector silencing by epigenetic modifications of integrated provirus. Gene Ther 2008, 15:49-53.

34. Nightingale SJ, Hollis RP, Pepper KA, Petersen D, Yu XJ, Yang C, Bahner I, Kohn DB: Transient gene expression by nonintegrating lentiviral vectors. Mol Ther 2006, I 3: I I 2I-I| 32 .

35. Apolonia L, Waddington SN, Fernandes C, Ward NJ, Bouma G, Blundell MP, Thrasher AJ, Collins MK, Philpott NJ: Stable gene transfer to muscle using non-integrating lentiviral vectors. Mol Ther 2007, I 5:1947-1954.

36. Yusufzai TM, Tagami H, Nakatani Y, Felsenfeld G: CTCF tethers an insulator to subnuclear sites, suggesting shared insulator mechanisms across species. Mol Cell 2004, 13:291-298.

37. Yahata K, Maeshima K, Sone T, Ando T, Okabe M, Imamoto N, Imamoto F: cHS4 insulator-mediated alleviation of promoter interference during cell-based expression of tandemly associated transgenes. J Mol Biol 2007, 374:580-590.

38. Ciuffi A, Mitchell RS, Hoffmann C, Leipzig J, Shinn P, Ecker JR, Bushman FD: Integration site selection by HIV-based vectors in dividing and growth-arrested IMR-90 lung fibroblasts. Mol Ther 2006, I3:366-373.

39. Mitchell RS, Beitzel BF, Schroder AR, Shinn P, Chen H, Berry CC, Ecker JR, Bushman FD: Retroviral DNA integration: ASLV, HIV, and MLV show distinct target site preferences. PLOS Biol 2004 2:E234.

40. Montini E, Cesana D, Schmidt M, Sanvito F, Ponzoni M, Bartholomae C, Sergi LS, Benedicenti F, Ambrosi A, Di SC, Doglioni C, Von KC, Naldini L: Hematopoietic stem cell gene transfer in a tumorprone mouse model uncovers low genotoxicity of lentiviral vector integration. Nat Biotechnol 2006, 24:687-696. 
4I. Lois C, Hong EJ, Pease S, Brown EJ, Baltimore D: Germline transmission and tissue-specific expression of transgenes delivered by lentiviral vectors. Science 2002, 295:868-872.

42. Park F: Lentiviral vectors: are they the future of animal transgenesis? Physiol Genomics 2007, 31:159-173.

43. Mirkovitch J, Mirault ME, Laemmli UK: Organization of the higher-order chromatin loop: specific DNA attachment sites on nuclear scaffold. Cell 1984, 39:223-232.

44. Heng HH, Goetze S, Ye C], Liu G, Stevens JB, Bremer SW, Wykes SM, Bode J, Krawetz SA: Chromatin loops are selectively anchored using scaffold/matrix-attachment regions. J Cell Sci 2004, I 1 7:999-1008.

45. Taher TE, Tulone C, Fatah R, D'Acquisto F, Gould DJ, Mageed RA: Repopulation of $B$-lymphocytes with restricted gene expression using haematopoietic stem cells engineered with lentiviral vectors. Gene Ther 2008, I5:998-1006.

46. Park F, Ohashi K, Kay MA: The effect of age on hepatic gene transfer with self-inactivating lentiviral vectors in vivo. Mol Ther 2003, 8:314-323.

47. Ma Y, Ramezani A, Lewis R, Hawley RG, Thomson JA: High-level sustained transgene expression in human embryonic stem cells using lentiviral vectors. Stem Cells 2003, 21: I I I-I I7.

48. Harraghy N, Gaussin A, Mermod N: Sustained Transgene Expression Using MAR Elements. Curr Gene Ther 2008, 8:353-366.

49. Sastry L, Xu Y, Cooper R, Pollok K, Cornetta K: Evaluation of plasmid DNA removal from lentiviral vectors by benzonase treatment. Hum Gene Ther 2004, I 5:22I-226.

50. Rosenqvist N, Hard Af SC, Samuelsson C, Johansen J, Lundberg C Activation of silenced transgene expression in neural precursor cell lines by inhibitors of histone deacetylation. J Gene Med 2002, 4:248-257.

51. Pfaffl MW: A new mathematical model for relative quantification in real-time RT-PCR. Nucleic Acids Res 200I, 29:e45.

Publish with Bio Med Central and every scientist can read your work free of charge

"BioMed Central will be the most significant development for disseminating the results of biomedical research in our lifetime. "

Sir Paul Nurse, Cancer Research UK

Your research papers will be:

- available free of charge to the entire biomedical community

- peer reviewed and published immediately upon acceptance

- cited in PubMed and archived on PubMed Central

- yours - you keep the copyright
BioMedcentral 